\title{
A framework to implement AI-integrated chatbot in educational institutes
}

\author{
Babpu Debnath \\ Aparna Agarwal
}

\author{
Middle East College \\ Middle East College
}

\begin{abstract}
The purpose of this paper is to explore the usefulness of chatbot in educational institutes such as schools and colleges and to propose a chatbot development plan that meet the needs. Usually chatbots are built for one specific purpose, for example, to answer general queries prospective students might have regarding admission. This paper aims to provide an artificialintelligence(AI) integrated chatbot framework that can help develop a multi-use chatbot. Study is based highly on qualitative data collected from case studies and journal articles. Primary data is also collected from interviews and questionnaires presented to appropriate staffs and students in college, in this case, Middle East College. Integrating AI into the chatbot to make it self-reliant, intelligent and learn from user interaction is necessary to make it deal with multiple fields. This requires complex algorithms, database management and extensive labor, thus making it very costly. However, if developed, this single chatbot can help students, faculties and other staffs greatly, not just as an assistant in answering frequently asked questions, but also in learning and teaching. The chatbot can be integrated with mobile app making it a part of daily life. Due to over complexity, the chatbot will first developed for use in one field then gradually expanded to other. A chatbot built for multiple purposes certainly holds more complexity than a single general purpose chatbot. This being said, having the software developed and tested in real life would have helped to better understand its flexibility and functionality.
\end{abstract}

\section{Introduction}

A chatbot is a software often integrated with artificial intelligence(AI) to simulate a human in text or voice interaction. Chatbots are generally built for specific tasks such as to virtually assist someone or to help people navigate daily life, etc. Although chatbots have come a long way since first developed, they are yet to pass the Turing test (Bradesko \& Mladenic, 2012). Nevertheless, the fact that they can temporarily deal with simple human tasks can greatly reduce overheads for companies and organizations by reducing the need to hire additional employees to do the job. Moreover, employees can have more time to focus on complex tasks, thus increasing productivity and efficiency (Rouse, 2019; V-Soft Consulting, n.d.).

Based on how chatbots operate, they can be classified into several types. Most chatbots are domain specific and developed for a specific need. For example, a chatbot might be developed to handle queries a prospective student might have before joining a university. Based on searches made, rarely if any study has been done to develop a multi-use chatbot that is capable of helping user in multiple sectors. That is, for instance, a chatbot developed for educational institute is capable of guiding people who might have any queries regarding admission, assist students in navigating through courses and help teachers in teaching and learning. However, building a multi-purpose chatbot can add to existing complexity, especially when attempting to integrate AI into the system to enable it to learn and evolve overtime. This sets a roadmap into first exploring the benefits of multi-purpose chatbot in educational institutes, user's expectations from these chatbots, proposing a shallow outline for a dynamically responsive multi-purpose chatbot and lastly to comprehend the potential barriers. 


\section{Study Design and Purpose}

Literatures based on the functionality of chatbots, their designs, usefulness and obstacles that hinder development of a chatbot have been reviewed. Additionally, interviews were conducted with a few faculty of Middle East College (MEC) to find their expectations from a multiuse chatbots. The paper is constructed based on these literature reviews and survey with the aim to support developers who wish to develop a multi-use chatbot for an educational institute. The finding from the study are presented as follows.

\section{Literature Review}

Colace (2018) and others had proposed a prototype of a Chatbot in the field of education. It had been developed to aid the students' learning for some specific subjects. The emphasis was mainly on the communication and the selection of the appropriate responses. For detecting the questions natural language processing (NLP) techniques were used and the domain ontologies were used to select the answers.

Gonda (2019) and others demonstrated in their work the chatbot that they implemented to improve the content delivery for a group assessment in Higher Education could address only five out of seven principles stated by Chickering and Gamson's. They said that the most difficult part in developing the chatbot was to train the chatbot for responses.

\section{Exploring the benefits for students and teachers}

A study conducted by Brandtzaeg and Følstad (2017) found that people prefer to use chatbot because it maximizes productivity, acts as a source of entertainment and increases curiosity regarding its reply. Their study also found that users tend to form social bondage with the chatbot thus making them want to use it. Applying this in context of education, Sjöström and his team (2018) assures that chatbots can provide instant answers to learners and make educational flow more interactive and dynamic. Chatbots can also significantly help students in learning in terms of memory retention and learning outcomes when compared to students who self-learn by using search engines (Abbasi \& Kazi, 2014). Chatbots can also be developed to understand learning styles of users and respond to their feedbacks which can also encourage trust, improve bondage, and motivate users. Additionally, Molnár and Szüts (2018) found from their study that students tend to believe chat mates more than a search engine, thus emphasizing the importance of chatbots in education. According to Cui (2015) MOOC's (Massive Open Online Courses) consists of three components - the lectures, quizzes and the peer tutors. MOOC'S have already had a positive global impact as it is revolutionizing the way students learn (Agarwal 2013). Thus chatbot in education can therefore be thought as an automated tutor that solves a learner's queries.

\section{Barriers to confront}

The communication between humans and chatbots are not so natural and requires further research to improve (Sjöström et al., 2018). According to Rahman, Mamun and Islam (2017), the primary issue when developing a dynamically responsive (answers queries which are not predefined by developer by searching knowledge base) chatbot is to enable chatbot to process natural language effectively. Additionally, the programming complexity due to use of AI machine learning algorithms restrict the efficient functionality of chatbots. The authors also mention in their study that there is insufficient input to feed chatbots to correctly answer the user. However, for the latter, Huang, Zhou and Yang (2007) proved that it is possible to extract question-answer pairs from online discussion forums using a cascaded framework. Based on set conditions so as to not gather irrelevant or poor quality answers, the chatbot was able to extract from 2,000 threads within two minutes. The sheer volume of information extracted can boost up the chatbot's knowledge base 
more quickly than any human even can.

Abdul-Kader and Woods (2015) reviewed and analyzed nine studies that affect chatbot design. The authors believe that chatbots are unable to improve at an affective pace due to not having common design practices among developers. This is because researchers often work in isolation and tend not to disclose any improved techniques they find. The author adds to the point that additional research and a need for common solution is required. Regarding the conversational user interfaces (CUI) Fadhil and Schiavo (2019) believes that messaging application are most popular worldwide due to their simple CUI. In their study, the authors showed that there is "no standardized way to represent all dialogs or a large range of conversational systems." Depending on the application, certain CUI is more versatile over others and has to be chosen carefully. They also point to the findings that having a human to verify the dialogue can hinder the repetitiveness in dialogues.

\section{Features of the chatbot based on survey findings}

Survey showed that few people were concerned that there will be loss of job in teaching and other sectors if a chatbot with multiple usage is introduced. However, majority of interviewees wished to have the chatbot integrated with a mobile application that can be accessed with ease. In MEC most services (e.g. students' attendance records, library books' catalogue, etc.) are digitized and can be accessed via web portal. Nevertheless, they argued that a chatbot which is aware of user (faculty/admin/student etc.) should provide answers to their queries in real time. A student who wish to know if a book is available in library can simply text the chatbot and get instant reply. Student and teachers should be able to ask study related questions to the chatbot and get tailored answers based on user. Faculty who wish to know scheduled date of an assessment need not check the course calendar, but simply text the chatbot. Likewise, a prospective student who does not have a user ID can question the chatbot accessible via MEC's website as a guest user and get answers to it. The chatbot should be able to hand over to appropriate staff if in case it is unable to answer. Among all, probably the most important feature demanded was to enable certain people to know the most frequently asked questions to the chatbot. Using the information teachers can know what part of subject students are unclear about. This can help the teacher focus on it and make teaching time more worthwhile. 


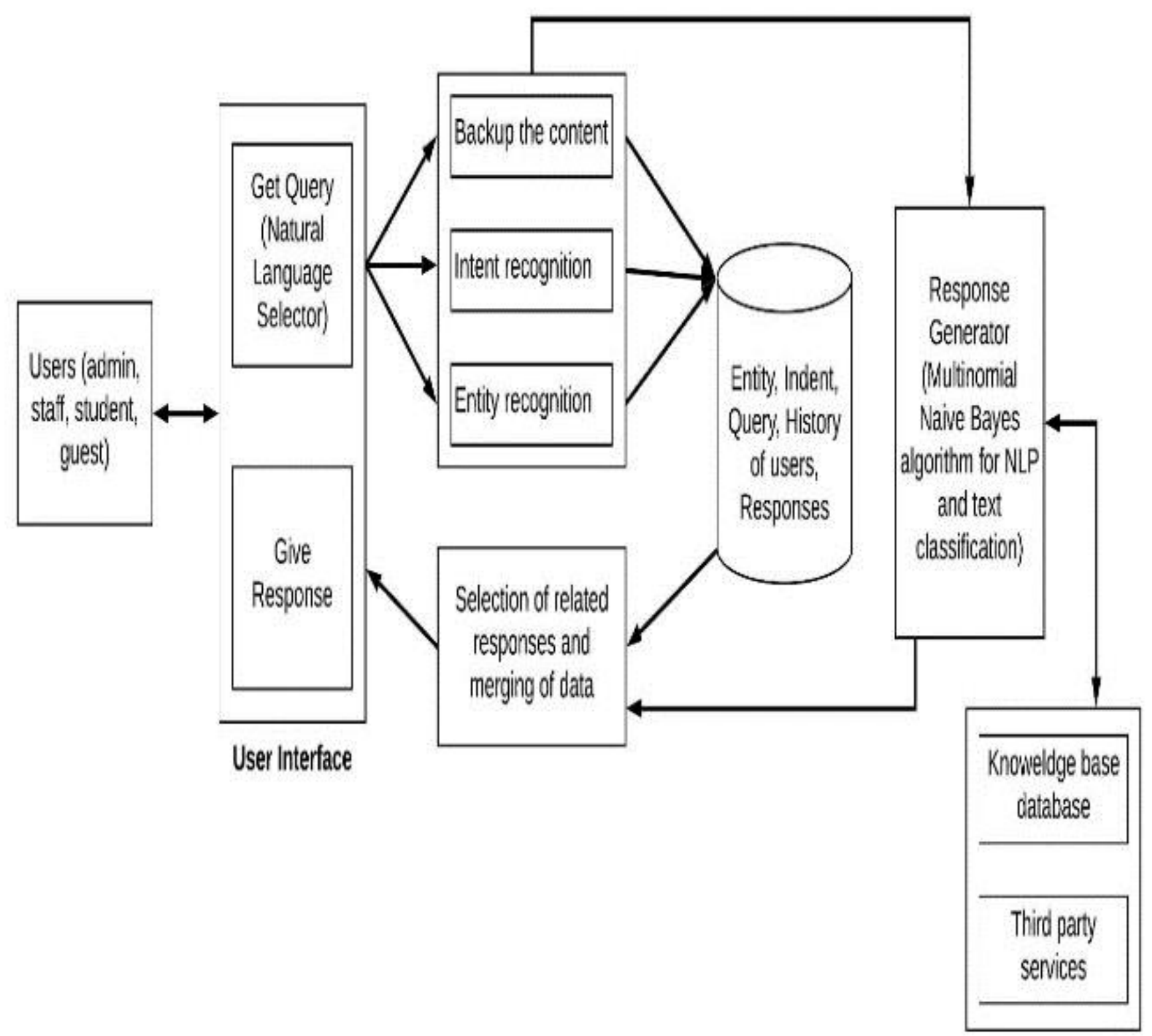

\section{Figure 1. The chatbot architecture}

Figure 1.

From the interview it is clear that there is a high expectation from a multiuse chatbot where the demand is to make almost all services easily accessible from one application. Based on finding from survey and literature reviewed, the following points are noted that needs to be considered when developing a multi-purpose chatbot for educational institute.

- A simplified CUI for the chatbot. Ensure that dialogues keep the user engaged (Fadhil and Schiavo, 2019).

- Dynamic chatbot with machine learning algorithms that can answer questions which are not predefined by searching knowledge base (Abbasi and Kazi, 2014).

- Define algorithm to search for answers from third party sources, i.e. discussion forums, to 


\section{Journal of Student Research}

Fourth Middle East College Student Research Conference, Muscat, Sultanate of Oman

aid students and teachers in education (Huang, Zhou and Yang, 2007).

- Keep log of users' activity. This can help teachers and other responsible personnel.

- Have access to user's details (user role, any course information etc.) to provide tailored results.

\section{The chatbot Architecture}

Figure 1 shows a general outline of how chatbot works. User communicates with the chatbot through user interface. This can be using graphics, text-based, audio based or a mixture. The interface gets the query and feed it to Natural Language Processor (NLP) which decodes the message and matches with a list of queries it supports. The NLP has two key components: Intent and Entity recognition. The first tries to identity the user's intent and matches with the intents it supports while the latter extracts key words in user's input. Since every user has signed in to use the chatbot, the chatbot is aware of the user type. This helps chatbot answer certain queries only for a particular user type. For example, a chatbot should reply "Cannot disclose information!" or "I am not aware of it." if a student asks "When is the next faculty meeting date?". A user can also sign in as Guest and get answers to queries such as admission fees for a particular Major. The option of signing in as Guest is to enable users without ID who wishes to know about the institute (e.g. a prospective student). Information access to these users are restricted accordingly. The dialogue management component of the system should be developed so as to keep user engaged and not feel that they are chatting to a machine. Chatbot replies like, "Never heard of that. What is it related to?" can help maintain user's engagement. Additionally, where required the chatbot should clarify with user so as to not process incorrect command, e.g. "You mean change code to 1025, right?".

The conversation will be backed up/recorded so that the chatbot can continue conversation later with user related to topic previously discussed, perhaps few hours/days ago. The chat log can also help teachers monitor the most asked question to chatbot regarding a particular course. This can aid teachers in providing a better education. The chatbot will occasionally ask for user's feedback. If the user provides a positive feedback, some data which addresses the path followed to provide user's satisfaction will be saved to knowledge base. Overtime, it will mine the backed up data to search for most user satisfied path and build up knowledge base to provide better user satisfaction. Backup of data will also help the chatbot to save answers to most commonly asked questions.

The knowledge base of the chatbot is to be built through automated machine learning algorithms and manual teaching by humans. Manually, people expert in specific domain should provide a list of possible questions and answers. The chatbot can then match key words in user's questions and provide the predefined answers. To expand the chatbot's capability, experts can feed additional documents and link the chatbot to domain specific third party services (such as discussion forums) from where the chatbot can self-learn and mine for answers to queries that are not pre-set. Here again, based on user's feedback, the chatbot can decide whether or not to save the chatbot's response to the query. If unable to find any answer or if the feedback is negative, the chatbot should hand over the task to appropriate staff. The staff can then reply user's query or feed the chatbot with that answer.

\section{Limitations}

Since the survey was conducted within MEC, most of the answers were tailored to needs of people working within the institute. A wider array of data was therefore not taken into consideration. Additionally, the chatbot architecture presented here needs to be tested for usefulness and efficiency before being implemented.

\section{Conclusion}




\section{Journal of Student Research}

Fourth Middle East College Student Research Conference, Muscat, Sultanate of Oman

Although chatbots have developed greatly over the past decades, there is still a need to develop the human-machine interaction and the ability to form semantically accurate meaningful sentences by analysing the input data. Public also needs be educated about the purpose of having a chatbot and understand that in education chatbots are not meant to replace teachers, but improve communication' between learner and teacher (Sjöström et al., 2018).

With the work presented above, the authors hope that developers can know what to expect when developing a multi-purpose chatbot system for educational institute and probably find a simplified guideline that can be followed during the development process. Since this article addresses some of the weaknesses and research gaps in chatbot development, a scholar can use this to seek and explore as yet undiscovered area or probably propose a better, more efficient design for developing a multi-purpose chatbot.

\section{Acknowledgment}

The authors would love to thank all the staffs of Middle Easy College who gave the opportunity to interview them.

\section{References}

Abbasi, S. \& Kazi, H. (2014). Measuring effectiveness of learning chatbot systems on Student's learning outcome and memory retention. Asian Journal of Applied Science and Engineering, 3, 57-66. doi: 10.15590/ajase/2014/v3i7/53576

Abbasi, S. \& Kazi, H. (2014). Measuring effectiveness of learning chatbot systems on Student's learning outcome and memory retention. Asian Journal of Applied Science and Engineering, 3, 57-66. doi: 10.15590/ajase/2014/v3i7/53576

Abdul-Kader, S. A. \& Woods, J. (2015). Survey on Chatbot Design Techniques in Speech Conversation Systems. International Journal of Advanced Computer Science and Applications, 6(7), 72-80. Retrieved from

https://thesai.org/Downloads/Volume6No7/Paper_12-Survey_on_Chatbot_Design_Techniques_in_Spe ech_Conversation_Systems.pdf

Agarwal, A. (Speaker). (2013). Why massive open online courses (still) matter [Video]. Edinburgh, Scotland: TEDGlobal 2013. Retrieved from https://www.ted.com/talks/anant_agarwal_why_massive_ open_online_courses_still_matter?utm_source=tedcomshare\&utm_medium=email\&utm_campaign= tedspread

Bradesko, L., \& Mladenic, D. (2012). A Survey of Chabot Systems through a Loebner Prize Competition. Retrieved from

https://pdfs.semanticscholar.org/9447/1160f13e9771df3199b3684e085729110428.pdf?.ga=2.1088 $\underline{68619.847311103 .1574207030-206774130.1574207030}$

Brandtzaeg, P.B. \& Følstad A. (2017). Why People Use Chatbots. In: Kompatsiaris I. et al. (Eds.), Internet Science. INSCI 2017. Lecture Notes in Computer Science (pp 377-392). Springer, Cham. Retrieved from https://doi.org/10.1007/978-3-319-70284-1_30

Colace, F., Santo, M. D., Lombardi, M., Pascale, F., Pietrosanto, A. \& Lemma, S. (2018) Chatbot for E-Learning: A Case of Study. International Journal of Mechanical Engineering and Robotics Research, 7(5), 528-533. doi: 10.18178/ijmerr.7.5.528-533

Cui, A. (2015, February 20). Massive Open Online Courses (MOOCs) and the next generation [Video file]. Retrieved from https://www.youtube.com/watch?v=gbkeWebvW1M 


\section{Journal of Student Research}

Fourth Middle East College Student Research Conference, Muscat, Sultanate of Oman

Fadhil, A. \& Schiavo, G. (2019). Designing for Health Chatbots. ArXiv, abs/1902.09022.

Gonda, D. E., Luo, J., Wong, Y. and Lei, C. (2019). Evaluation of Developing Educational Chatbots Based on the Seven Principles for Good Teaching. 2018 IEEE International Conference on Teaching, Assessment, and Learning for Engineering (TALE) (pp. 446-453). Wollongong, NSW, Australia. doi: 10.1109/TALE.2018.8615175

Huang, J., Zhou, M. \& Yang, D. (2007). Extracting Chatbot Knowledge from Online Discussion Forums. IJCAI 2007, Proceedings of the 20th International Joint Conference on Artificial Intelligence (pp. 423-428). Hyderabad, India. Retrieved from https://www.ijcai.org/Proceedings/07/Papers/066.pdf

Molnár, G. \& Szüts, Z. (2018). The Role of Chatbots in Formal Education. IEEE 16th International Symposium on Intelligent Systems and Informatics (pp. 197-201). Subotica, Serbia. doi: 10.1109/SISY.2018.8524609

Rahman, AM., Mamun, AA \& Islam, A. (2017). Programming challenges of chatbot: Current and future prospective. 2017 IEEE Region 10 Humanitarian Technology Conference (pp. 75-78). Dhaka, Bangladesh: IEEE. doi: 10.1109/R10-HTC.2017.8288910

Rouse, M. (2019). Chatbot. Retrieved 20 November, 2019 from https://searchcustomerexperience.techtarget.com/definition/chatbot

Sjöström, J., Aghaee, N., Dahlin, M. \& Ågerfalk, P. J. (2018). Designing Chatbots for Higher Education Practice. International Conference on Information Systems Education and Research (pp. 1-10). San Francisco, CA. Retrieved from https://www.researchgate.net/publication/328245964_Designing_Chatbots_for_Higher_Education_P ractice

V-Soft Consulting. (n.d.). Understanding The Conversational Chatbot Architecture. Retrieved 22 November, 2019 from https://blog.vsoftconsulting.com/blog/understanding-the-architecture-ofconversational-chatbot 